\title{
Optic neuropathies in childhood: a review of etiology and treatment
}

\author{
Ceren Günbey, Bahadır Konuşkan \\ Division of Pediatric Neurology, Hacettepe University Faculty of Medicine, Ankara, Turkey. \\ E-mail: cerengunbey06@gmail.com \\ Received: 20th November 2018, Revised: 27th December 2018, Accepted: 30th January 2019
}

\begin{abstract}
SUMMARY: Günbey C, Konuşkan B. Optic neuropathies in childhood: a review of etiology and treatment. Turk J Pediatr 2019; 61: 471-476.

Optic neuropathy (ON) is a common problem in childhood resulting from various causes including ischemic, metabolic, hereditary, traumatic and inflammatory events. The latter represents the most prevalent group; the term "optic neuritis" is generally used for this group which can manifest as part of a systemic inflammatory disease, a central nervous system disorder, or as an isolated entity. The aim of this review is to highlight the clinical characteristics and treatments of $\mathrm{ON}$ for pediatricians, focusing on optic neuritis.
\end{abstract}

Key words: optic neuropathy, children, vision loss, optic neuritis, treatment.

Congenital or acquired problems of vision are common throughout childhood. Moreover, they can constitute the initial or only manifestation of a systemic disease. Therefore, evaluation of visual acuity and visual fields must be part of the routine pediatric examination. In infants and toddlers, drawing the baby's attention to the examiner's face or to a toy and then moving the target slowly will allow the assessment of visual ability, particularly if each eye is tested separately. After age 4, visual acuity can be screened with standard tools. Visual fields of the young child can be tested by bringing a toy from the periphery and observing the child's reaction when the object enters his/her visual field. A relative afferent pupillary defect is an important sign for optic neuropathy.

Detailed examination of the fundus, performed by an ophthalmologist, gives significant clues for differential diagnosis between retinal causes of vision loss and optic neuropathy $(\mathrm{ON})$, also between subgroups of ON. Disc swelling with hemorrhages may point to ischemic or inflammatory disease, whereas peripapillary retinal telangiectasia suggests Leber hereditary ON..$^{1,2}$ The disc may appear normal in retrobulbar $\mathrm{ON}$. In these cases, a contrast enhanced magnetic resonance imaging (MRI) of both orbita and brain should be performed. ${ }^{3}$ Optical coherence tomography to document peripapillary nerve fiber layer thickness and visual evoked potentials to measure optic pathways' conduction are other useful tests.

Impairment of vision due to disturbance of the optic nerve is defined as ON. While this term is often used interchangeably with optic neuritis, the latter should be confined to infectious, postinfectious or inflammatory conditions. ON in childhood results from various causes which will be reviewed below with emphasis on the largest etiological group, inflammatory ON, or optic neuritis (Table I).

\section{Ischemic Optic Neuropathies}

Insufficient blood supply to the optic nerve causes ischemic ON. Although rare in children, it is reported as a complication of chronic peritoneal dialysis, major cardiovascular or spinal surgical interventions, or drugs (e.g., sildenafil given for heart disease) ${ }^{4,5}$ More local vascular causes like Takayasu arteritis, ChurgStrauss syndrome, and sickle cell disease are other possible underlying disorders. ${ }^{6,7}$ The classical finding, altitudinal visual field defect, is seldom described by children. There is no specific treatment for ischemic ON. Case reports show poor visual outcome in children. ${ }^{8}$ 
Table I. Etiology of Optic Neuropaties in Children.

\begin{tabular}{l}
\hline Etiological Groups \\
\hline Ischemic \\
Metabolic \\
- Toxic \\
Nereditary \\
$\quad$ Leber hereditary optic neuropathy \\
- Dominant optic atrophy \\
Traumatic \\
Inflammatory \\
1- Infectious and parainfectious \\
2- Demyelinating \\
: Isolated optic neuropathy \\
- Nhronic relapsing inflammatory optic neuropathy \\
- Associated with multiple sclerosis \\
- Associated with acute disseminated encephalomyelitis
\end{tabular}

\section{Metabolic Optic Neuropathies}

Toxic agents and nutritional deficiencies can cause metabolic $\mathrm{ON}$ by disrupting pathways of energy production or oxidative stress regulation. Main causes of nutritionalmetabolic $\mathrm{ON}$ in childhood are deficiencies of vitamins $\mathrm{A}, \mathrm{B} 12$ and the biotinidase enzyme. Toxic ON is caused by agents like linezolid, ethambutol, or amiodarone. ${ }^{9-11}$ Treatment relies on replacing the specific nutrient and eliminating the toxic agent whenever possible. Factors affecting the prognosis are the visual acuity at initial examination and the delay before diagnosis and intervention.

\section{Hereditary Optic Neuropathies}

Leber hereditary ON (LHON) is a mitochondrial disorder that can cause bilateral, severe visual impairment. Young children and adults, predominantly males, are affected. Mitochondrial DNA mutations at nucleotide position $11778,3460,14484$ are the most common mutations; their combined prevalence was found as $1 / 45000$ in Europe. ${ }^{12}$ Typically, subacute painless visual loss begins in one eye and the other eye is affected over weeks to months. ${ }^{13}$ Severe and permanent visual impairment, such as a visual acuity of 20/200 or worse, can develop in many patients during the course of LHON. ${ }^{14}$ Idebenone, a synthetic analog of ubiquinone, is recommended in early stages of the disease. ${ }^{15}$
Dominant optic atrophy (DOA) is another inherited bilateral $\mathrm{ON}$ starting in the first two decades and progressing throughout adulthood. Its prevalence ranges between $1 / 1.200-1.50 .000 .{ }^{16}$ It is caused by mutations in the OPA1 gene which encodes a mitochondrial enzyme. Patients suffer from slowly progressive visual loss, but final visual acuity usually remains better than 20/200 in both eyes. Some patients also show extraocular manifestations of mitochondrial disease. Given the absence of any proven treatment, idebenone may be considered. ${ }^{17}$

\section{Traumatic Optic Neuropathies}

Traumatic $\mathrm{ON}$ is a rare cause of vision loss that results from direct, or more frequently, indirect injury to the optic nerve, which is observed among $2.5-12.2 \%$ of head and face trauma cases. ${ }^{18}$ Its annual incidence in the pediatric age group is around 1/1.000.000 with a male predominance. Treatment with steroids and surgical decompression of the optic nerve have been tried alone or in combination, with no proven efficacy. Final visual acuity varies and is influenced mainly by visual acuity at initial examination after the trauma. ${ }^{19}$

\section{Inflammatory Optic Neuropathies}

The term "optic neuritis" is widely used for this group which can manifest as part of a systemic inflammatory disease or as a separate 
entity. Isolated bilateral optic neuritis in childhood should be considered as a central nervous system disease. Infection-related and demyelinating $\mathrm{ON}$ are also included in this category because demyelination is usually accompanied by inflammation in childhood, as supported by steroid responsiveness.

Clinical findings are acute/subacute visual impairment, periocular pain, and swelling of the optic disc, usually bilateral in young children. Underlying disorders are various and visual recovery is favorable in the pediatric population..$^{20,21}$

\section{1- Infectious and Parainfectious Optic Neuropathies}

This group constitutes the most common causes of visual impairment in the pediatric age group. It can occur as a single episode or as recurrent attacks, both either isolated or associated with other systemic diseases. It can be part of infectious diseases via direct invasion or secondary inflammation in viral infections such as Epstein-Barr, cytomegalovirus, rubella, human immunodeficiency virus (HIV), varicella; bacterial ones by Mycoplasma pneumonia, Coxiella burnetii, Borrelia burgdorferi, Brucella species, Treponema pallidum, or Toxocara species. ${ }^{22-25}$ A global health problem, tuberculosis, may also cause $\mathrm{ON}$, with or without concomitant meningitis. ${ }^{26}$

Infections with enterovirus, mumps, rubella or simple upper respiratory tract viruses, and vaccination with hepatitis $B$, influenza, measles-mumps-rubella, tetanus vaccines may cause post-infectious and post-immunisation ON via inflammatory mechanisms. ${ }^{27-31}$

\section{2- Demyelinating ON:}

Inflammation leading to demyelination in the optic nerve may present as isolated $\mathrm{ON}$ or as part of demyelinating disorders as described below. $^{32,33}$ Inflammatory demyelination of the central nervous system may be associated with serum IgG antibodies against myelin oligodendrocyte glycoprotein (MOG-IgG), and this antibody has become a biomarker describing a subgroup with favorable outcome in children. A retrospective study of 14 children with optic neuritis followed up for mean 6 years resulted in final diagnoses of isolated optic neuritis in $8(57 \%)$, recurrent optic neuritis in 2 (14\%), multiple sclerosis (MS) in 2 (14\%), Sjögren's syndrome in 1 (7\%) and neuromyelitis optica in 1 (7\%). ${ }^{34}$ Another recent large series of children with isolated optic neuritis followed-up for median 4 years revealed diagnoses of MS in $145(40.6 \%)$, single isolated optic neuritis in $167(46.7 \%)$, recurrent isolated optic neuritis in $40(11.2 \%)$ and neuromyelitis optica in $5(1.4 \%){ }^{35}$

Neuromyelitis optica-asssociated optic neuritis: Neuromyelitis optica is a relatively rare disease characterized by single or recurrent inflammatory attacks involving optic nerves and spinal cord. Autoantibodies against the water channel aquaporin-4 (AQP4) play a role in the pathogenesis. ${ }^{36,37}$ The term "neuromyelitis optica spectrum disorder" adopted in recent years comprises seronegative patients presenting with optic neuritis, myelitis, encephalopathy or brain stem symptoms and signs. ${ }^{38}$ Prognosis is variable and recurrences are expected. ${ }^{37}$

Optic neuritis associated with multiple sclerosis (MS): The diagnosis of MS is based on both clinical and radiological features that were recently revised in children. ${ }^{39}$ Optic neuritis may be the first clinical presentation in MS or may be seen during the relapsingremitting course of MS. The visual prognosis is favorable except in one recent series where vision loss was found to be more frequent in MS compared to other causes of ON..$^{40,41}$

Optic neuritis associated with acute disseminated encephalomyelitis (ADEM): ADEM, an inflammatory demyelinating syndrome with multiple neurologic symptoms and typical MRI findings, is more common than MS in children. ${ }^{39,42}$ Unilateral or bilateral optic neuritis can be part of, and even the only clinical manifestation of, ADEM. The visual outcome is favorable. ${ }^{20,42}$

Chronic relapsing inflammatory ON (CRION): First described in 2003, this group became smaller after the description of AQP4-Ab and MOG IgG-positive groups. Its characteristic features are unilateral or bilateral painful ON that relapses upon withdrawal of steroid treatment. ${ }^{43,44}$ Young adults (youngest age: 14 years) and females are predominantly affected. 
The incidence is unknown, and the diagnosis is clinical as there is no serological biomarker. Cranial and spinal imaging is normal, ruling MS and neuromyelitis optica spectrum disorder out.

\section{Treatment Options for ON}

\section{Acute period}

Whether isolated or part of neurological or systemic disease, ON needs prompt attention because of the importance of the visual function and the risk of rapid atrophy of the edematous optic nerve compressed in the optic canal. Treatment targets the etiologic factors. Toxic and metabolic conditions are to be corrected rapidly. In LHON, mitochondrial toxins should be avoided; idebenone is being used despite the absence of clear evidence. No effective treatment exists for traumatic and ischemic $\mathrm{ON}$, although steroids have been administered in anecdotal reports. Inflammatory $\mathrm{ON}$, the largest group in children, usually benefits from steroid and immunomodulatory treatment as described below.

\section{Corticosteroids}

The Optic Neuritis Treatment Trial has been one of the most important studies that focused on usage of steroids. It showed intravenous methylprednisolone (1 gr/day for 3 days) followed by short-term low-dose oral prednisolone accelerates visual recovery. It may also decrease the risk of being diagnosed with MS in two years' follow-up. ${ }^{45,46}$ Although no pediatric guidelines have been published yet, intravenous methylprednisolone $(30 \mathrm{mg} /$ $\mathrm{kg}$ /day; maximum $1 \mathrm{~g}$ /day) for 3 to 5 days followed by $1 \mathrm{mg} / \mathrm{kg} /$ day oral steroid tapered over in 1-1.5 months constitutes the generally preferred treatment in children. ${ }^{47}$ In a recent Cochrane review, no effect of corticosteroid treatment was reported on final visual acuity in optic neuritis. ${ }^{48}$

\section{Intravenous immunoglobulin}

Intravenous immunoglobulin (IVIg) may possess a protective effect on neurons. Its administration in steroid-refractory $\mathrm{ON}$ cases is being debated, as publications exist both favoring or opposing its effect on visual acuity and preventing relapses in ON. ${ }^{47,49,50}$ Part of the discrepancy may result from different timing, dosing or assessment, and also from the underlying etiology and risk factors. Although not subjected to randomized controlled trials in pediatric optic neuritis, IVIg may also be an option as second-line treatment $(400 \mathrm{mg} / \mathrm{kg} /$ day for 5 days for single or repeated rounds) in children.

\section{Plasmapheresis}

Therapeutic plasma exchange or immunoadsorption are other possible secondline options to modify the immune system via removal of pathogenic antibodies in optic neuritis. The effect of plasmapheresis in central nervous system inflammatory demyelinating diseases, especially in steroid-resistant MS in adults, is well known. Though those studies examine MS, ADEM and neuromyelitis optica spectrum disorder but not isolated optic neuritis, and pediatric studies are scarce, plasmapheresis should be considered in cases with persistent severe visual impairment after corticosteroid therapy. ${ }^{51,52}$ A recent study showed a positive effect of plasma exchange on visual recovery in 34 adults with isolated idiopathic optic neuritis. ${ }^{53}$

\section{New Treatments}

Neuroprotective agents like erythropoietin, phenytoin, or amiloride, and remyelinating ones like an antibody against leucinerich repeat and immunoglobulin domaincontaining protein (LINGO-1) are explored as therapeutic options. ${ }^{54,55}$

Optic neuritis in childhood is primarily a systemic pediatric problem. Etiological approach to the history, physical examination, neurological findings and paraclinical investigations are within the responsibility of the pediatrician who also conducts the treatment and follow-up with the ophthalmologist.

\section{REFERENCES}

1. Biousse V, Newman NJ. Ischemic optic neuropathies. N Engl J Med 2015; 372: 2428-2436.

2. Behbehani R. Clinical approach to optic neuropathies. Clin Ophthalmol 2007; 1: 233-246. 
3. The clinical profile of optic neuritis. Experience of the Optic Neuritis Treatment Trial. Optic Neuritis Study Group. Arch Ophthalmol 1991; 109: 1673-1678.

4. Lapeyraque AL, Haddad E, Andre JL, et al. Sudden blindness caused by anterior ischemic optic neuropathy in 5 children on continuous peritoneal dialysis. Am J Kidney Dis 2003; 42: E3-E9.

5. Kim JS, Deputy S, Vives MT, Aviles DH. Sudden blindness in a child with end-stage renal disease. Pediatr Nephrol 2004; 19: 691-693.

6. Matalia J, Kasturi N, Anaspure HD, Shetty BK. Tonic pupil, anterior ischemic optic neuropathy in a teenager with Takayasu arteritis. Can J Ophthalmol 2013; 48: e159-e163.

7. Partal A, Moshfeghi DM, Alcorn D. Churg-Strauss syndrome in a child: retina and optic nerve findings. Br J Ophthalmol 2004; 88: 971-972.

8. Di Zazzo G, Guzzo I, De Galasso L, et al. Anterior ischemic optical neuropathy in children on chronic peritoneal dialysis: report of 7 cases. Perit Dial Int 2015; 35: 135-139.

9. Hayati AA, Wan-Hitam WH, Cheong MT, Yunus R, Shatriah I. Optic neuritis in a child with biotinidase deficiency: case report and literature review. Clin Ophthalmol 2012; 6: 389-395.

10. McAbee GN, Prieto DM, Kirby J, Santilli AM, Setty R. Permanent visual loss due to dietary vitamin A deficiency in an autistic adolescent. J Child Neurol 2009; 24: 1288-1289.

11. Van Stavern GP. Metabolic, hereditary, traumatic, and neoplastic optic neuropathies. Continuum (Minneap Minn) 2014; 20 (4 Neuro-ophthalmology): 877-906.

12. Mascialino B, Leinonen M, Meier T. Meta-analysis of the prevalence of Leber hereditary optic neuropathy mtDNA mutations in Europe. Eur J Ophthalmol 2012; 22: 461-465.

13. Newman NJ, Biousse V, Newman SA, et al. Progression of visual field defects in leber hereditary optic neuropathy: experience of the LHON treatment trial. Am J Ophthalmol 2006; 141: 1061-1067.

14. Meyerson C, Van Stavern G, McClelland C. Leber hereditary optic neuropathy: current perspectives. Clin Ophthalmol 2015; 9: 1165-1176.

15. Carelli V, Carbonelli M, de Coo IF, et al. International Consensus Statement on the Clinical and Therapeutic Management of Leber Hereditary Optic Neuropathy. J Neuroophthalmol 2017; 37: 371-381.

16. Skidd PM, Lessell S, Cestari DM. Autosomal dominant hereditary optic neuropathy (ADOA): a review of the genetics and clinical manifestations of ADOA and ADOA+. Semin Ophthalmol 2013; 28: 422-426.

17. Barboni P, Valentino ML, La Morgia C, et al. Idebenone treatment in patients with OPA1-mutant dominant optic atrophy. Brain 2013; 136 (Pt 2): e231.

18. Van Stavern GP, Biousse V, Lynn MJ, Simon DJ, Newman NJ. Neuro-ophthalmic manifestations of head trauma. J Neuroophthalmol 2001; 21: 112-117.
19. Ford RL, Lee V, Xing W, Bunce C. A 2-year prospective surveillance of pediatric traumatic optic neuropathy in the United Kingdom. J AAPOS 2012; 16: 413-417.

20. Cakmakli G, Kurne A, Guven A, et al. Childhood optic neuritis: the pediatric neurologist's perspective. Eur J Paediatr Neurol 2009; 13: 452-457.

21. Wilejto M, Shroff M, Buncic JR, Kennedy J, Goia C, Banwell B. The clinical features, MRI findings, and outcome of optic neuritis in children. Neurology 2006; 67: 258-262.

22. Pahor D. Bilateral optic neuritis in a child following Epstein-Barr virus infection. Coll Antropol 2005; 29(Suppl 1): 129-132.

23. Mansor AM, Li HK. Cytomegalovirus optic neuritis: characteristics, therapy and survival. Ophthalmologica 1995; 209: 260-266.

24. Choi SY, Choi YJ, Choi JH, Choi KD. Isolated optic neuritis associated with Mycoplasma pneumoniae infection: report of two cases and literature review. Neurol Sci 2017; 38: 1323-1327.

25. Ahn SJ, Ryoo NK, Woo SJ. Ocular toxocariasis: clinical features, diagnosis, treatment, and prevention. Asia Pac Allergy 2014; 4: 134-141.

26. Venkatesh P, Garg SP, Verma L, et al. Combined optic neuropathy and central retinal artery occlusion in miliary tuberculosis. Retina 2001; 21: 375-377.

27. Moradian S, Ahmadieh H. Early onset optic neuritis following measles-rubella vaccination. J Ophthalmic Vis Res 2008; 3: 118-122.

28. Erguven M, Guven S, Akyuz U, Bilgiç O, Laloglu F. Optic neuritis following hepatitis B vaccination in a 9-year-old girl. J Chin Med Assoc 2009; 72: 594-597.

29. Sugita K, Ando M, Minamitani K, Miyamoto H, Niimi $\mathrm{H}$. Magnetic resonance imaging in a case of mumps postinfectious encephalitis with asymptomatic optic neuritis. Eur J Pediatr 1991; 150: 773-775.

30. Farris BK, Pickard DJ. Bilateral postinfectious optic neuritis and intravenous steroid therapy in children. Ophthalmology 1990; 97: 339-345.

31. Topaloglu H, Berker M, Kansu T, Saatci U, Renda Y. Optic neuritis and myelitis after booster tetanus toxoid vaccination. Lancet 1992; 339: 178-179.

32. Dos Passos GR, Oliveira LM, da Costa BK, et al. MOG-IgG-associated optic neuritis, encephalitis, and myelitis: lessons learned from neuromyelitis optica spectrum disorder. Front Neurol 2018; 9: 217.

33. Carnero Contentti E, De Virgiliis M, Hryb JP, et al. Aquaporin-4 serostatus and visual outcomes in clinically isolated acute optic neuritis. J Neuroophthalmol 2019; 39: 165-169.

34. Lee CG, Lee B, Lee J, Lee M. The natural course of clinically isolated syndrome in pediatric patients. Brain Dev 2015; 37: 432-438. 
35. Heussinger N, Kontopantelis E, Gburek-Augustat J, et al; for GRACE-MS (German-speaking Research Alliance for ChildrEn with Multiple Sclerosis). Oligoclonal bands predict multiple sclerosis in children with optic neuritis. Ann Neurol 2015; 77: 1076-1082.

36. Drori T, Chapman J. Diagnosis and classification of neuromyelitis optica (Devic's syndrome). Autoimmun Rev 2014; 13: 531-533.

37. Tenembaum S, Chitnis T, Nakashima I, et al. Neuromyelitis optica spectrum disorders in children and adolescents. Neurology 2016; 87(9 Suppl 2): S59-S66.

38. Wingerchuk DM, Banwell B, Bennett JL, et al; International Panel for NMO Diagnosis. International consensus diagnostic criteria for neuromyelitis optica spectrum disorders. Neurology 2015; 85: 177-189.

39. Krupp LB, Tardieu M, Amato MP, et al; International Pediatric Multiple Sclerosis Study Group. International Pediatric Multiple Sclerosis Study Group criteria for pediatric multiple sclerosis and immune-mediated central nervous system demyelinating disorders: revisions to the 2007 definitions. Mult Scler 2013; 19: 1261-1267.

40. Waldman A, Ness J, Pohl D, et al. Pediatric multiple sclerosis: Clinical features and outcome. Neurology 2016; 87 (9 Suppl 2): S74-S81.

41. Averseng-Peaureaux D, Mizzi M, Colineaux H, et al; Kidbiosep Study Group. Paediatric optic neuritis: factors leading to unfavourable outcome and relapses. Br J Ophthalmol 2018; 102: 808-813.

42. Dale RC, de Sousa C, Chong WK, Cox TC, Harding B, Neville BG. Acute disseminated encephalomyelitis, multiphasic disseminated encephalomyelitis and multiple sclerosis in children. Brain 2000; $123(\mathrm{Pt}$ 12): 2407-2422.

43. Kidd D, Burton B, Plant GT, Graham EM. Chronic relapsing inflammatory optic neuropathy (CRION). Brain 2003; 126(Pt 2): 276-284.

44. Petzold A, Plant GT. Chronic relapsing inflammatory optic neuropathy: a systematic review of 122 cases reported. J Neurol 2014; 261: 17-26.
45. Beck RW, Cleary PA, Anderson MM Jr, et al; The Optic Neuritis Study Group. A randomized, controlled trial of corticosteroids in the treatment of acute optic neuritis. N Engl J Med 1992; 326: 581-588.

46. Beck RW, Cleary PA, Trobe JD, et al; The Optic Neuritis Study Group. The effect of corticosteroids for acute optic neuritis on the subsequent development of multiple sclerosis. N Engl J Med 1993; 329: 17641769 .

47. Collinge JE, Sprunger DT. Update in pediatric optic neuritis. Curr Opin Ophthalmol 2013; 24: 448-452.

48. Gal RL, Vedula SS, Beck R. Corticosteroids for treating optic neuritis. Cochrane Database Syst Rev 2015; 8: CD001430.

49. Viswanathan S, Wong AH, Quek AM, Yuki N. Intravenous immunoglobulin may reduce relapse frequency in neuromyelitis optica. J Neuroimmunol 2015; 282: 92-96.

50. Roed HG, Langkilde A, Sellebjerg F, et al. A doubleblind, randomized trial of IV immunoglobulin treatment in acute optic neuritis. Neurology 2005; 64: 804-810

51. Koziolek M, Mühlhausen J, Friede T, et al. Therapeutic apheresis in pediatric patients with acute CNS inflammatory demyelinating disease. Blood Purif 2013; 36: 92-97.

52. Jacquet C, Garnier A, Cheuret E. Plasma exchange and immunoadsorption in pediatric inflammatory optic neuropathy resistant to corticosteroid therapy: Four French cases. Neuropediatrics 2016; 47: 175178.

53. Deschamps R, Gueguen A, Parquet N, et al. Plasma exchange response in 34 patients with severe optic neuritis. J Neurol 2016; 263: 883-887.

54. Black JA, Liu S, Hains BC, Saab CY, Waxman SG Long-term protection of central axons with phenytoin in monophasic and chronic-relapsing EAE. Brain 2006; 129 (Pt 12): 3196-3208.

55. Lo AC, Saab CY, Black JA, Waxman SG. Phenytoin protects spinal cord axons and preserves axonal conduction and neurological function in a model of neuroinflammation in vivo. J Neurophysiol 2003; 90: 3566-3571. 